\title{
Energy efficient routing and task scheduling for autonomous transfer vehicles in intra logistics
}

\author{
Inci Saricicek ${ }^{1, *}$, Sinem Bozkurt Keser ${ }^{2}{ }^{*}$, Azmi Cibi $^{2}$, Tahir Ozdemir ${ }^{2}$, Ahmet Yazici $^{2}$ \\ ${ }^{1}$ Dept. of Industrial Engineering, Eskisehir Osmangazi University \\ Eskişehir, Turkey \\ ${ }^{2}$ Dept. of Computer Engineering, Eskisehir Osmangazi University \\ Eskişehir, Turkey \\ *Corresponding author: sbozkurt@ogu.edu.tr
}

\begin{abstract}
Autonomous Transfer Vehicles (ATVs) are becoming increasingly prevalent in intra logistics. Industry 4.0 is bringing us closer to the efficient routing and scheduling of autonomous multi robot systems which perform transportation tasks. In this study, an energy efficient routing and scheduling system is proposed to minimize the total energy that the vehicles spend. Not only travelled distance but also the load of the vehicle is considered between two points. The routes of vehicles are obtained by using the proposed Hybrid Simulated Annealing Algorithm. An algorithm for the initial solution is also proposed for determining of the minimum number of vehicles for pickup and delivery requests. The performance of the algorithm is compared with the best solutions of the test problems in the literature. Besides, the proposed energy efficient routing and task scheduling model is compared with the classical distance model for routing and scheduling with backhauls. An analysis of trade-offs between energy and distance is proposed for intra logistics.
\end{abstract}

Keywords: Autonomous Transfer Vehicles; energy efficient routing; hybrid simulated annealing; pickup and delivery tasks; Vehicle Routing Problem with backhauls.

\section{Introduction}

The routing and scheduling of a fleet of vehicles is one of the most common problems in logistics. The prevalence of transportation operations combined with the large variety of real-world constraints and characteristics have created a multitude of vehicle routing problems (VRP). There are many subclasses of the VRP problems (Eksioglu et al., 2009). The Pickup and Delivery Problem (PDP) is one of them. A classification of the PDP is given by Lin et al. (2014). Empty vehicle travelling is called deadheading and this situation causes increasing the logistics cost. To reduce the deadheading, vehicles visit the delivery points first and then pickup finished products at pickup points and back to the depot. This activity is called backhauling. VRP with backhauling where for each individual route all deliveries have to be made before the first pickup is one of the possible variants in intra logistics (Goetschalckx, 2011). Material handling vehicles such as Automated Guided Vehicle (AGV), forklift, etc. are used for intra logistics. Recently, Autonomous Transfer Vehicles (ATVs) have been replaced by classic AGVs. The vehicles are electrically operated and periodically charged. ATVs have the features of sensing features in the environment and navigating autonomously without any limitations on the route (Xidias et al., 2009).

ATVs allow more flexible production systems and material handling systems in factories. There are several studies about mission planning, task planning or routing of ATVs in smart factories. Emilio et al. 
(2002) proposes a randomized path planning approach for autonomous vehicles. Liu et al. (2006) utilize an improved ant colony algorithm to plan the motion path for each robot. As it is aforementioned, the main objective is to minimize the travel distance. Xidias et al. (2009) propose an algorithm to solve a vehicle scheduling and routing problem in 2D industrial environments. A modified Genetic Algorithm is utilized to minimize the distance. Herrero-Pérez \& Martínez-Barberá (2010) utilize decentralized navigation control and a Distributed Petri Net to model and control a flexible material handling system. It consists of autonomous vehicles that are appropriate for flexible manufacturing systems. Hussein $e t$ al. (2012) compare three different metaheuristics such as Tabu Search, Simulated Annealing, and Genetic Algorithm that minimize the total travelled distance. In the experiments, Simulated Annealing outperforms other algorithms in terms of the computation times but Tabu Search provides the best path. Xidias et al. (2016) present a modified Genetic Algorithm to solve the task scheduling problem of a fleet of autonomous vehicles. Chávez et al. (2016) propose a Pareto ant colony algorithm for multi-depot VRPB to minimize distance, travel time and energy consumption. Magdy et al. (2017) apply various metaheuristics algorithms in order to solve the path planning problem of autonomous vehicles. Xidias (2018) uses the modified Genetic Algorithm to solve routing and task scheduling problem simultaneously by using the constructed matrix. Rahul (2020) proposes a co-evolutionary for very complex mission planning. Wang et al. (2019) propose a modified Genetic Algorithm for task allocation of AGVs by considering the remaining battery charge of each AGV.

The industrial sector's energy consumption has accounted for about $50 \%$ of the world's total energy consumption in the 2010's. It has almost doubled over the last 60 years (Fang et al., 2011). Fuel consumption of vehicles is dependent on a lot of factors in logistics. Minimizing the fuel consumption in VRPs is considered by Kara et al. (2007), Bektaş and Laporte (2011), Xiao et al. (2012) and Ghannadpour and Hooshfar (2016) in the literature. While it is aimed to reduce the fuel consumption for trucks in logistics, it is important to address pollution routing problem in shop floor transportation problems and reduce energy consumption of transport vehicles in intra logistics. While the body of literature in internal logistics is vast, to the best of our knowledge, there are no papers that address task scheduling of pickup and delivery requests with backhauls for autonomous vehicles and energy saving routing in production environments.

In this study, we studied routing and scheduling of pickup and delivery problem of ATVs that are electrically operated and periodically charged. Vehicle Routing Problem with Backhauls (VRPB) where an ATV starts the route from a depot, visits linehaul stations for delivery, and then visits backhaul stations for pickup, and returns to the depot is handled. We estimate energy consumption according to the load of vehicle and the distance travelled between two points. Hybrid Simulated Annealing (HSA) is proposed to an energy saving routing and task scheduling. An heuristic algorithm for the initial solution that minimize the number of vehicles is also suggested. The proposed energy saving routing and scheduling algorithm minimizes consumed energy by considering distance travelled and the loads of ATVs for each pickup and delivery point. The algorithm is compared with the classical distance travelled model for routing and scheduling with backhauls to evaluate the trade-offs. The main contributions of the paper are as follows:

- VRPB is discussed in intra logistics for electrically operated ATVs.

- Path planning, scheduling and routing problems are evaluated in terms of energy savings.

- The number of ATV for requests is minimized.

The rest of the paper is structured as follows: In the following section, the problem description, proposed energy saving routing and task scheduling model, proposed initial solution algorithm, and proposed hybrid simulated annealing algorithm are explained. In section 3, experimental results for the proposed algorithm are given. The last section includes the conclusion of the study. 


\section{Routing and task scheduling of pickup and delivery tasks with backhauls}

ATVs are expected to increase the flexibility and efficiency in intra logistics of production. Thus, the need for methods that provide effective solutions in a short time for task planning of ATVs becomes important. In our industrial environment, ATVs take the parts from Depot1 to the respective workstations in the direction of the pickup and delivery requests from the workstations in the production line as seen in Fig 1. Then they will take the products or semi-products from the pickup points and will deliver them to Depot2. Without loss of generality, each workstation has pickup (P) and delivery (D) points in the production environment.

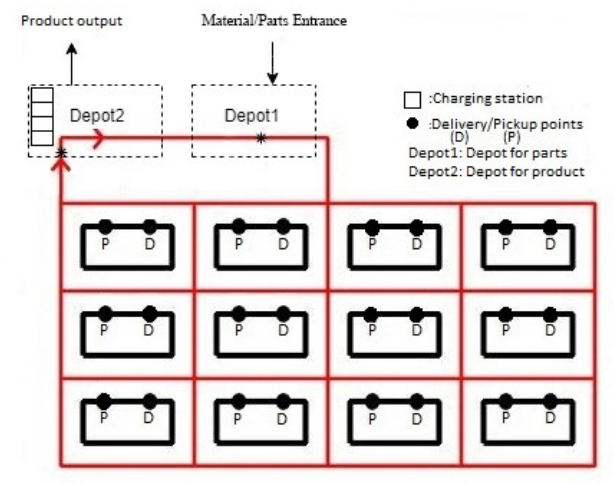

Fig. 1. The covered 2D layout of the factory environment.

The proposed routing and task scheduling algorithm for VRPB consists of the following steps:

Step 1: Modeling of problem environment.

Step 2: Initial solution generation by minimizing the number of ATV.

Step 3: Hybrid Simulated Annealing Algorithm for routing and task scheduling.

The details of each step are given in the following subsections.

\subsection{Modeling of Energy-Saving Routing and Task Scheduling}

The model takes into consideration of the number of ATVs, their starting positions, and transportation requests, and the graph of the environment. It generates routes for the ATVs by minimizing the total energy consumed.

\section{Assumptions:}

There are enough ATVs to complete all the tasks in the environment.

The speed of the ATVs varies according to their load.

The weight of the no-load ATV, $w_{v_{j}}$, is $120 \mathrm{~kg}$, and each has a maximum loading capacity represented by $V C_{\max }, 80 \mathrm{~kg}$.

More than one ATV can perform pickup and delivery tasks for each station.

Pickup and delivery tasks can be divided.

Indices:

$i, j$ : Index for nodes

$k$ : Index for ATVs

Parameters:

$d_{i j}$ : Distance between node $i$ and $j$

$\rho^{*}$ : Energy spent when the ATV is full-load

$\rho_{0}$ : Energy spent when the ATV is no-load 
$V C_{\max }$ : ATV load capacity

Decision variables:

$$
x_{i j k}=\left\{\begin{array}{c}
1 ; \text { ATV } k \text { moves from node } i \text { to } j \\
0 ; \text { otherwise }
\end{array}\right.
$$

$y_{i j k}$ : load from node $i$ to node $j$ with ATV $k$

Objective function:

$$
\begin{gathered}
\operatorname{Min} z=\sum_{i \in V} \sum_{j \in V} \sum_{k \in K} d_{i j}\left(\rho_{0} x_{i j k}+\alpha y_{i j k}\right) \\
\alpha=\left(\rho^{*}-\rho_{0}\right) / V C_{\max }
\end{gathered}
$$

The objective function in Equation 1 shows the total energy minimized that depends on the transported load and travelled distance between nodes. $\alpha$ gives the average energy consumption per unit load. Total energy consumed by the partially loaded ATVs is calculated by multiplying decision variable $y_{i j k}$ with $\alpha$.

Based on the number of ATVs, their starting positions, and the received transportation requests, the assignment of requests and appropriate routes for the ATVs are obtained in order to minimize total energy consumed by the ATVs. The conventional routing and scheduling problem is known as a combinational optimization problem, which is an NP-hard problem and cannot be solved by existing exact algorithms in reasonable time. Therefore, metaheuristics algorithms are generally used in order to solve these kinds of problems. In this study, HSA is proposed to solve the routing and task scheduling problem of ATVs.

\subsection{Proposed Initial Solution Algorithm}

A heuristic algorithm is proposed to generate an initial solution. The pseudocode of the algorithm is given in Algorithm 1.

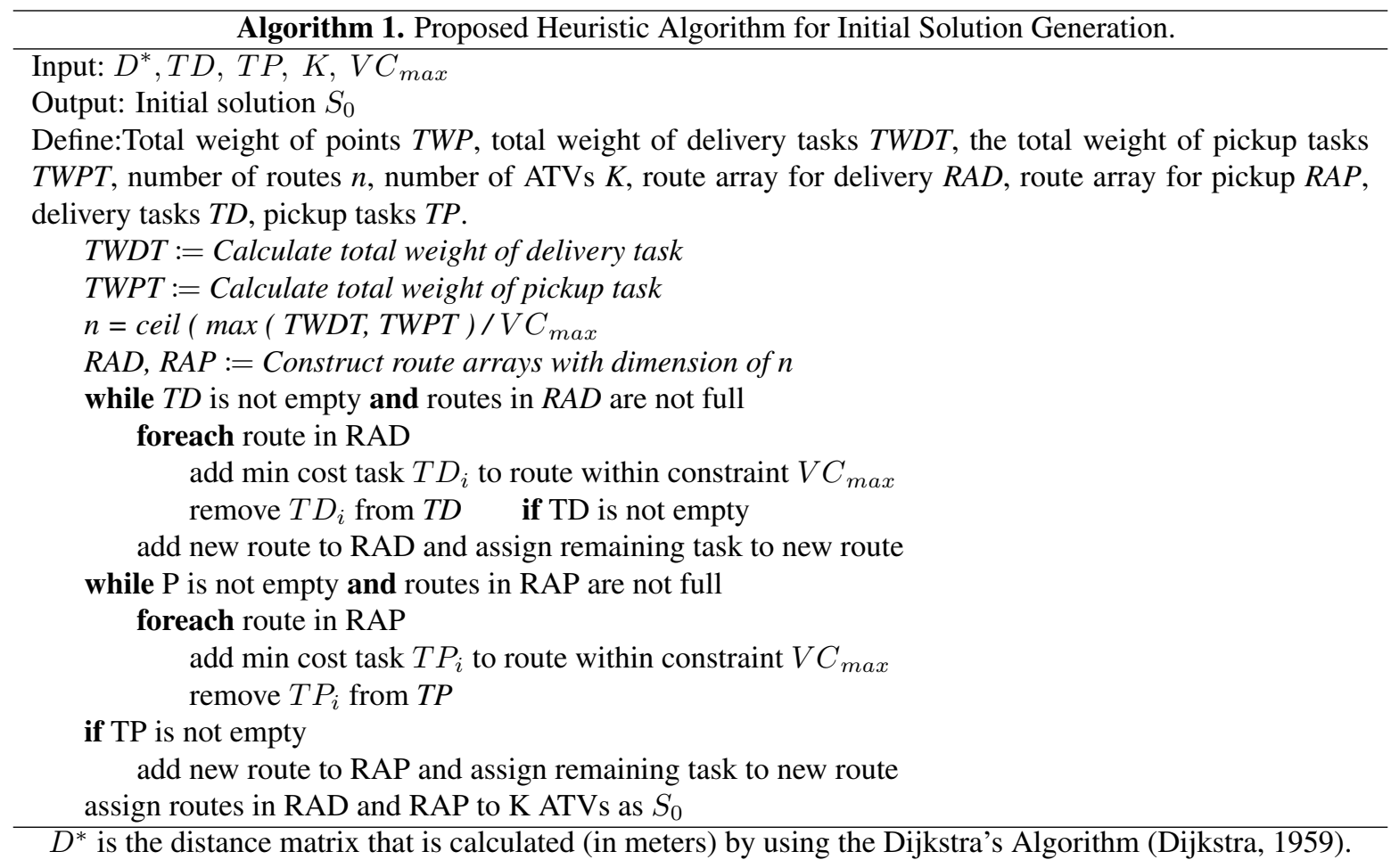

The algorithm finds the initial solution in three steps. In the first step, it finds the required number of routes to complete the given tasks by considering the ATV capacity. It classifies the pickup and delivery tasks by P/D node number, finds the total weight of points (TWP) for each product type at each P/D 
points, and sums these up to find the total weight of delivery tasks (TWDT) and the total weight of pickup tasks (TWPT). Then, it estimates the number of routes, $n$, to complete the given tasks. Note that each ATV is assumed to be homogenous in terms of the ATV capacity $V C$. In the second step of the algorithm, route arrays are created based on the costs of the tasks. The route array for delivery (RAD) is created based on the index, which is the ratio of distance to TWP. As a procedure, heavier products are delivered first. In the same way, the route array for pickup (RAP) is created based on the index, which is Distance $\times T W P$. As a procedure, lighter products are picked up first. In the last step of the initial solution, the route arrays are assigned to available ATVs considering the number of ATVs, $K$. Note that the number of ATV, $K$, and the number of routes, $n$, are not necessarily equal. If $K$ is equal to $n$, then each route is assigned to an ATV. If $K$ is less than $n$, then the ATVs cannot complete all tasks in the first tour, and $(n-K)$ routes are assigned to ATVs for the second tour.

A list of tasks for delivery and pickup are generated for a sample problem in the Table 1 . The delivery point 29 requests three different items with a total quantity of 37.

Table 1. List of tasks for delivery and pickup.

\begin{tabular}{|c|c|c|c|c|c|c|}
\hline \# Items & Point number & Type of the task (P/D) & Item & Quantity & Weight(kg) & TWP(kg) \\
\hline \multirow{3}{*}{3 Items } & 29 & Delivery & $\mathrm{A}$ & 2 & 1 & 2 \\
\hline & 29 & Delivery & $\mathrm{D}$ & 2 & 10 & 20 \\
\hline & 29 & Delivery & $\mathrm{E}$ & 1 & 15 & 15 \\
\hline \multirow{3}{*}{3 Items } & 37 & Delivery & $\mathrm{A}$ & 3 & 1 & 3 \\
\hline & 37 & Delivery & $\mathrm{C}$ & 1 & 7 & 7 \\
\hline & 37 & Delivery & $\mathrm{E}$ & 2 & 15 & 30 \\
\hline \multirow{3}{*}{3 Items } & 31 & Delivery & $\mathrm{A}$ & 5 & 1 & 5 \\
\hline & 31 & Delivery & $\mathrm{C}$ & 2 & 7 & 14 \\
\hline & 31 & Delivery & $\mathrm{F}$ & 1 & 25 & 25 \\
\hline \multirow{3}{*}{3 Items } & 39 & Delivery & $\mathrm{B}$ & 2 & 4 & 8 \\
\hline & 39 & Delivery & $\mathrm{D}$ & 2 & 10 & 20 \\
\hline & 39 & Delivery & $\mathrm{F}$ & 1 & 25 & 25 \\
\hline \multirow{2}{*}{2 Items } & 41 & Delivery & B & 1 & 4 & 4 \\
\hline & 41 & Delivery & $\mathrm{C}$ & 1 & 7 & 7 \\
\hline \multirow{2}{*}{2 Items } & 24 & Pickup & $\mathrm{X}$ & 1 & 2 & 2 \\
\hline & 24 & Pickup & $\mathrm{Z}$ & 2 & 9 & 18 \\
\hline \multirow{3}{*}{3 Items } & 26 & Pickup & B & 1 & 4 & 4 \\
\hline & 26 & Pickup & $X$ & 1 & 2 & 2 \\
\hline & 26 & Pickup & $\mathrm{Y}$ & 1 & 5 & 5 \\
\hline \multirow{3}{*}{3 Items } & 28 & Pickup & $X$ & 5 & 2 & 10 \\
\hline & 28 & Pickup & $\mathrm{Z}$ & 2 & 9 & 18 \\
\hline & 28 & Pickup & $\mathrm{L}$ & 1 & 20 & 20 \\
\hline \multirow{3}{*}{3 Items } & 30 & Pickup & $X$ & 2 & 2 & 4 \\
\hline & 30 & Pickup & $\mathrm{Z}$ & 1 & 9 & 9 \\
\hline & 30 & Pickup & M & 1 & 25 & 25 \\
\hline \multirow{3}{*}{3 Items } & 36 & Pickup & $\mathrm{N}$ & 1 & 16 & 16 \\
\hline & 36 & Pickup & $\mathrm{Z}$ & 2 & 9 & 18 \\
\hline & 36 & Pickup & $\mathrm{L}$ & 1 & 20 & 20 \\
\hline \multirow{3}{*}{3 Items } & 38 & Pickup & $Y$ & 2 & 5 & 10 \\
\hline & 38 & Pickup & $\mathrm{Z}$ & 2 & 9 & 18 \\
\hline & 38 & Pickup & $X$ & 6 & 2 & 12 \\
\hline \multirow{2}{*}{2 Items } & 42 & Pickup & $\mathrm{A}$ & 2 & 1 & 2 \\
\hline & 42 & Pickup & $X$ & 1 & 2 & 2 \\
\hline
\end{tabular}

In the initial solution generation, pickup and delivery tasks are classified and TWP is calculated for each item by multiplying the quantity and weight. The minimum route number (n) that is required completing the tasks can be calculated as: 


$$
n=\left[\frac{\max (T W D T, T W P T)}{V C_{\max }}\right]
$$

\subsection{Proposed Hybrid Simulated Annealing Algorithm}

The proposed algorithm starts with an initial solution. An ATV can make multiple deliveries and multiple pickups by travelling to more than one P/D point. For a test problem with 3 ATVs and 12 points, the initial and the neighbor solutions are demonstrated in Table 2.

Table 2. Representation of initial and neighbour solutions.

\begin{tabular}{ccc}
\hline Route \# & Initial Solution & Neighbour Solution \\
\hline 1 & $37 \rightarrow 41 \rightarrow 31(1 ;$ item $) \rightarrow 42 \rightarrow 24 \rightarrow 36$ & $37 \rightarrow 41 \rightarrow 39(1$ item $) \rightarrow 42 \rightarrow 24 \rightarrow 36$ \\
2 & $39(3$ item $) \rightarrow 31(1$ item $) \rightarrow 30 \rightarrow 38$ & $39(2$ item $) \rightarrow 31(2$ item $) \rightarrow 30 \rightarrow 38$ \\
3 & $29 \rightarrow 31(1$ item $) \rightarrow 26 \rightarrow 28$ & $29 \rightarrow 31(1$ item $) \rightarrow 26 \rightarrow 28$ \\
\hline
\end{tabular}

As seen in Table 2, the pairwise interchange process for swap moves is used to generate neighborhoods. While generating neighborhoods, two random numbers are derived and two numbers are interchanged randomly. The neighbor solution is obtained by swapping 31 and 39 randomly in the initial solution.

Energy-saving routing and task scheduling for pickup and delivery with backhauls is achieved by using the proposed HSA. Pseudo-code of the HSA is given in Algorithm 2.

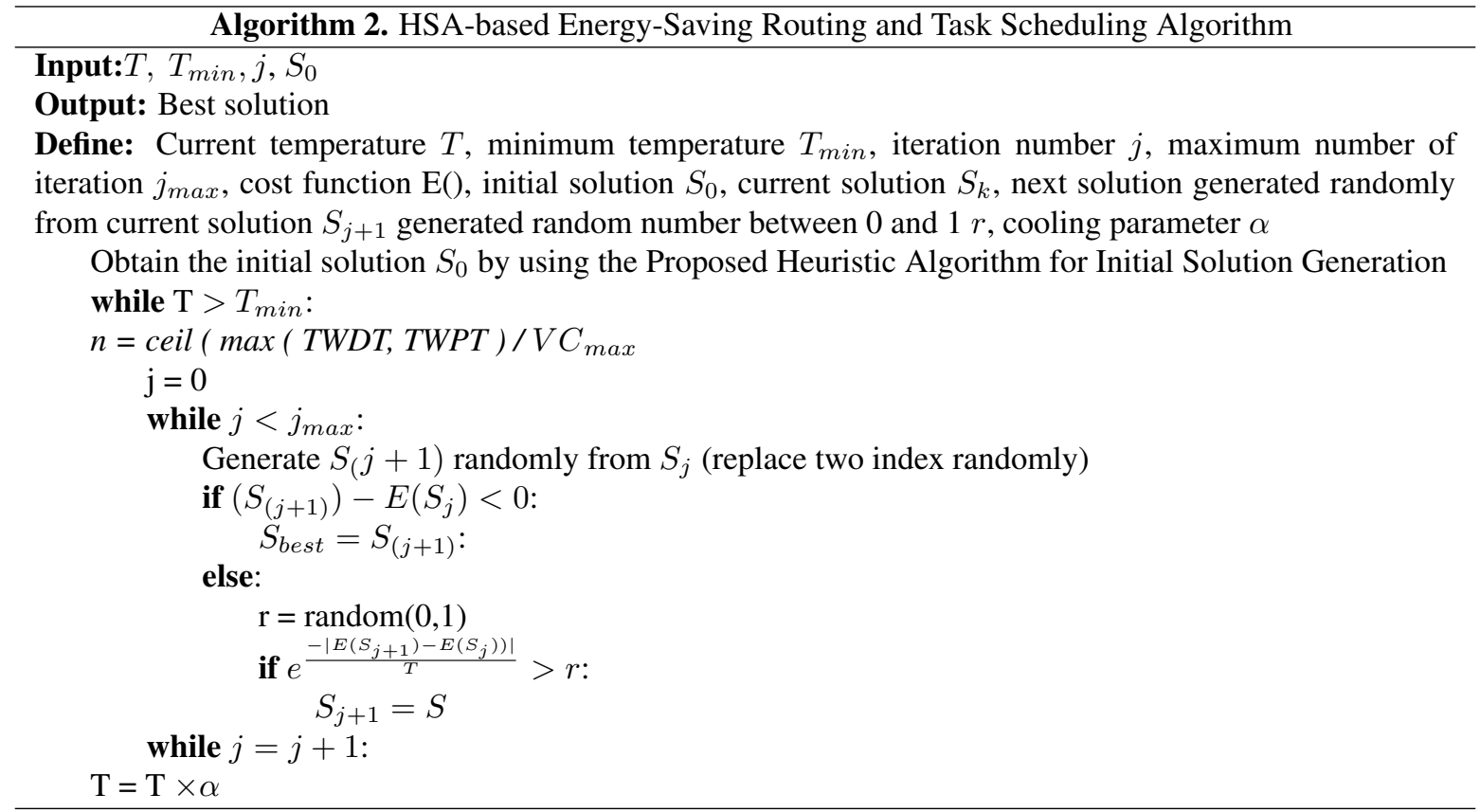

In the literature, the first set of problems for VRP with backhauls is originally proposed by Goetschalckx \& Jacobs-Blecha (1989). The performance of the proposed HSA is tested for 19 benchmark datasets and the results are shown in Table 3. In order to show the efficiency of the proposed algorithm, the results obtained for minimizing the total travel distance are compared with the best known values in the literature (Brandau, 2006). All experiments are executed on an 8-core Windows 10 workstation $(4 \times$ dual core $3.4 \mathrm{Ghz}$, Intel(R) Core(TM) i7-6700) with 8GB of RAM. The HSA is implemented by using MS C\#.NET under the .NET Framework 4.5 and MS Visual Studio 2015. The parameters of HSA is selected as $T=10000, T_{\min }=100, \alpha=0.9999, j_{\max }=0$.

The "best solution" column is the solution cost taken from Brandau (2006). The last column of Table 3 shows the gaps between the best values in literature and the proposed HSA. The optimal or near optimal results are obtained by using HSA for the problems in literature. 
Table 3. Comparison of the results of the proposed HSA algorithm with the best results in the literature for the traditional distance model.

\begin{tabular}{cccccccc}
\hline \multirow{2}{*}{ Problem\# $\begin{array}{c}\text { \# Pickup } \\
\text { Point }\end{array}$} & $\begin{array}{c}\text { \#Delivery } \\
\text { Point }\end{array}$ & $\begin{array}{c}\text { Vehicle } \\
\text { Capacity }\end{array}$ & \#Vehicles & $\begin{array}{c}\text { Optimum } \\
\text { Solution }\end{array}$ & HSA & Gap\% \\
\hline A1 & 20 & 5 & 1,550 & 8 & 229,886 & $\mathbf{2 2 9 , 8 8 6}$ & $0.00 \%$ \\
A2 & 20 & 5 & 2,550 & 5 & 180,119 & $\mathbf{1 8 0 , 1 1 9}$ & $0.00 \%$ \\
A3 & 20 & 5 & 4,050 & 4 & 163,405 & 164,787 & $0.85 \%$ \\
A4 & 20 & 5 & 4,050 & 3 & 155,796 & $\mathbf{1 5 5 , 7 9 6}$ & $0.00 \%$ \\
B1 & 20 & 10 & 1,600 & 7 & 239,080 & $\mathbf{2 3 9 , 0 8 6}$ & $0.00 \%$ \\
B2 & 20 & 10 & 2,600 & 5 & 198,048 & 198,433 & $0.19 \%$ \\
B3 & 20 & 10 & 4,000 & 3 & 169,372 & 170,670 & $0.77 \%$ \\
C1 & 20 & 20 & 1,800 & 7 & 249,448 & 256,576 & $2.86 \%$ \\
C2 & 20 & 20 & 2,600 & 5 & 215,020 & 217,764 & $1.28 \%$ \\
C3 & 20 & 20 & 4,150 & 5 & 199,346 & 203,144 & $1.91 \%$ \\
C4 & 20 & 20 & 4,150 & 4 & 195,366 & 200,494 & $2.62 \%$ \\
D1 & 30 & 8 & 1,700 & 12 & 322,530 & 325,252 & $0.84 \%$ \\
D2 & 30 & 8 & 1,700 & 11 & 316,709 & 317,288 & $0.18 \%$ \\
D3 & 30 & 8 & 2,750 & 7 & 239,479 & $\mathbf{2 3 9 , 4 7 9}$ & $0.00 \%$ \\
D4 & 30 & 8 & 4,075 & 5 & 205,832 & $\mathbf{2 0 5 , 8 3 2}$ & $0.00 \%$ \\
\hline
\end{tabular}

\section{Experimental results}

The HSA is implemented by using MS C\#.NET under the .NET Framework 4.5 and MS Visual Studio 2015. The proposed energy-saving model is compared to the traditional model which minimizes the total distance. The total distance travelled and the consumed energy are shown for both models in Table 4 by using HSA

Table 4. The comparison of the proposed energy-saving model with the traditional distance model.

\begin{tabular}{|c|c|c|c|c|c|}
\hline \multirow{3}{*}{$\begin{array}{l}\text { Number } \\
\text { of ATV }\end{array}$} & \multirow{3}{*}{$\begin{array}{l}\text { Assigned } \\
\text { Route }\end{array}$} & \multicolumn{2}{|c|}{ Traditional Distance Model } & \multicolumn{2}{|c|}{ Proposed Energy-Saving Model } \\
\hline & & \multicolumn{2}{|c|}{ Objective Function 1: Min Distance } & \multicolumn{2}{|c|}{ Objective Function 2: Min Energy } \\
\hline & & Distance $(\mathrm{m})$ & Energy ( kg.m ) & Distance $(m)$ & Energy ( kg.m ) \\
\hline 1 & 1 & 4,463 & 754,446 & 4,463 & 735,936 \\
\hline 2 & 2 & 4,272 & 735,238 & 4,272 & 726,676 \\
\hline 3 & 3 & 3,773 & 664,236 & 3,773 & 655,266 \\
\hline & 12,508 & $2,153,920$ & 12,508 & $2,117,878$ \\
\hline
\end{tabular}

As seen in Table 4, the distance and energy consumption are calculated as 3,773 $m$ and 664,236 $k g \times m$ by the traditional total-distance minimizing model with three ATVs. On the other hand, the values are achieved as 3,773 $\mathrm{m}$ and $655,266 \mathrm{~kg} \times \mathrm{m}$ for the distance and consumed energy, respectively. Although the proposed model has found the same distance value as the traditional model, $1.67 \%$ of the energy compared to the traditional model has been saved for three ATVs. The best routes according to the proposed model for each ATV are shown in Figure 2.

As seen in Figure 2 (a), the ATV starts its route from node 22 by taking the parts from Depot1. Since each ATV has to complete its delivery task first, the ATV visits the node 29. Then, the ATV goes back to the pickup node 28 . The ATV completes the pickup tasks after visiting node 26 and 24 . The ATV arrives at node 21 (Depot2) to deliver the products from the pickup points to Depot2.

In Figure 2 (b), the $2^{\text {nd }}$ ATV starts its route from node 22 (Depot1). It then follows node 41, 37, and 39 to complete its delivery tasks. Then, the ATV goes back to node 38 for the pickup task. The ATV completes the pickup tasks after visiting node 36 . Note that due to the capacity of the ATV, it can only 


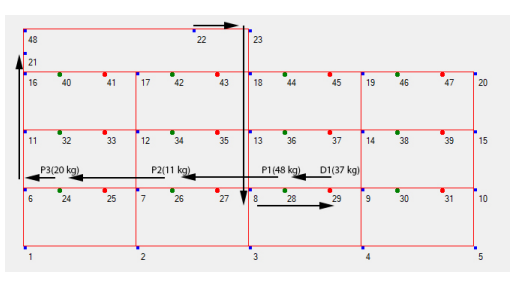

(a) Route of the 1st ATV

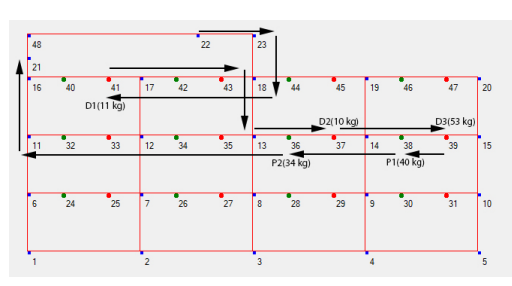

(b) Route of the 2nd ATV

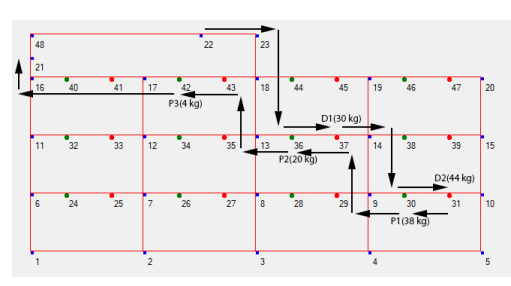

(c) Route of the 3rd ATV

Fig. 2. Route assignments of ATVs.

visit two pickup nodes in this trip. The ATV arrives at node 21 (Depot2) after finishing all its delivery and pickup tasks.

The $3^{\text {rd }}$ ATV starts its route from node 22 (Depot1) in Figure 2 (c). Then, the ATV follows node 37 and 31 to fulfill its delivery task. Then, the ATV visits node 30, 36, and 42 to complete all its pickup tasks. Finally, the ATV arrives at node 21 (Depot2) to deliver the products from the pickup points to Depot2.

In order to compare the models in the proposed algorithm, 20 different test problems are generated for the factory environment in Figure 1. The test problems involve heterogeneous loads with the minimum ATV requirement from 2 to 9 . Table 5 compares the results obtained by the traditional distance model with the results obtained by the proposed energy-saving model using the proposed HSA. The last column of this table shows the gaps between two models.

Table 5. The comparison of the proposed energy-saving model with the traditional distance model using proposed HSA algorithm.

\begin{tabular}{|c|c|c|c|c|c|c|c|c|c|}
\hline \multirow[b]{2}{*}{ Problem } & \multirow[b]{2}{*}{ \#ATV } & \multicolumn{3}{|c|}{ HSA with Traditional Distance Model } & \multicolumn{3}{|c|}{ HSA with Proposed Energy-Saving Model } & \multicolumn{2}{|c|}{ Saving } \\
\hline & & $\begin{array}{r}\text { Energy } \\
(\text { kg.m) }\end{array}$ & $\begin{array}{l}\text { Distance } \\
(m)\end{array}$ & $\begin{array}{l}\text { Computation } \\
\text { Time (s) }\end{array}$ & $\begin{array}{c}\text { Energy } \\
(\text { kg.m })\end{array}$ & $\begin{array}{l}\text { Distance } \\
(m)\end{array}$ & $\begin{array}{l}\text { Computation } \\
\text { Time (s) }\end{array}$ & $\begin{array}{c}\text { Energy } \\
(\text { kg.m })\end{array}$ & $\begin{array}{l}\text { Distance } \\
(m)\end{array}$ \\
\hline $1 \_20$ & 5 & $1,136,392$ & 6,533 & 11.60 & $1,124,546$ & 6,567 & 11.60 & 11,846 & -34 \\
\hline $2 \_20$ & 5 & 653,406 & 3,908 & 8.56 & 648,156 & 3,908 & 8.52 & 5,250 & 0 \\
\hline $3 \_20$ & 5 & $1,010,612$ & 5,989 & 11.05 & $1,006,112$ & 5,989 & 11.05 & 4,500 & 0 \\
\hline $4 \_20$ & 5 & 990,556 & 6,049 & 9.43 & 977,226 & 6,049 & 9.49 & 13,330 & 0 \\
\hline $5 \_20$ & 5 & 748,338 & 4,579 & 8.78 & 746,088 & 4,579 & 9.27 & 2,250 & 0 \\
\hline $6 \_20$ & 6 & 982,854 & 5,832 & 11.29 & 981,288 & 5,952 & 11.32 & 1,566 & -120 \\
\hline 7_20 & 5 & 707,526 & 4,309 & 8.17 & 696,946 & 4,309 & 8.23 & 10,580 & 0 \\
\hline 8_20 & 5 & 875,515 & 5,363 & 9.84 & 871,465 & 5,389 & 9.81 & 4,050 & -26 \\
\hline $9 \_20$ & 5 & $1,398,764$ & 7,981 & 13.73 & $1,384,700$ & 8,161 & 13.72 & 14,064 & -180 \\
\hline $10 \_20$ & 4 & 591,783 & 3,604 & 6.01 & 587,313 & 3,628 & 5.99 & 4,470 & -24 \\
\hline $11 \_20$ & 6 & 866,902 & 5,430 & 10.07 & 865,882 & 5,430 & 9.71 & 1,020 & 0 \\
\hline $12 \_20$ & 6 & 859,815 & 4,857 & 9.91 & 848,023 & 4,879 & 9.95 & 11,792 & -22 \\
\hline $13 \_20$ & 9 & $1,259,449$ & 7,328 & 11.53 & $1,254,679$ & 7,328 & 11.35 & 4,770 & 0 \\
\hline $14 \_20$ & 4 & 610,477 & 3,822 & 7.71 & 609245 & 3,822 & 7.70 & 1,232 & 0 \\
\hline $15 \_20$ & 2 & 499,870 & 2,944 & 5.46 & 490,410 & 3,034 & 5.54 & 9,460 & -90 \\
\hline $16 \_20$ & 7 & $1,006,106$ & 6,083 & 10.77 & $1,002,288$ & 6,083 & 10.66 & 3,818 & 0 \\
\hline $17 \_20$ & 3 & 562,160 & 3,349 & 7.03 & 552,820 & 3,349 & 6.92 & 9,340 & 0 \\
\hline 18_20 & 5 & $1,000,695$ & 5,641 & 10.22 & 983,179 & 5,761 & 10.14 & 17,516 & -120 \\
\hline $19 \_20$ & 5 & 990,428 & 5,967 & 10.97 & 975,710 & 5,967 & 10.87 & 14,718 & 0 \\
\hline \multirow[t]{3}{*}{$20 \_20$} & 7 & $1,148,179$ & 6,653 & 11.88 & $1,136,287$ & 6,683 & 11.90 & 11,892 & -30 \\
\hline & Total & $17,899,827$ & 106,221 & & $17,742,363$ & 106,867 & & 157,464 & -646 \\
\hline & Mean & $894,991.35$ & $5,311.05$ & & $887,118.15$ & $5,343.35$ & & 7,873.20 & -32.30 \\
\hline
\end{tabular}

As seen in the Table 5, total distance and energy consumption are calculated as 17,899,827 $\mathrm{m}$ and $106,221[\mathrm{~kg} \times \mathrm{m}]$ by the traditional total-distance minimizing model with three ATVs. On the other hand, the values are achieved as 17,742,363 $\mathrm{m}$ and 106,867 $[\mathrm{kg} \times \mathrm{m}]$ or the distance and consumed energy, respectively, by using the proposed energy-saving model. While the energy saving is $7,873[\mathrm{~kg}$ $\times m]$ in average, the total distance increases as $32.3 \mathrm{~m}$ in average for each route planning problem.

Although the proposed algorithm has found the same distance value as the traditional distance model, 
there is an energy saving in 11 out of 20 problem sets. While energy saving is occurred in all of the problems, the total distance increases in 9 out of 20 problems. Trade-offs between energy and distance are demonstrated for each problem in Figure 3.

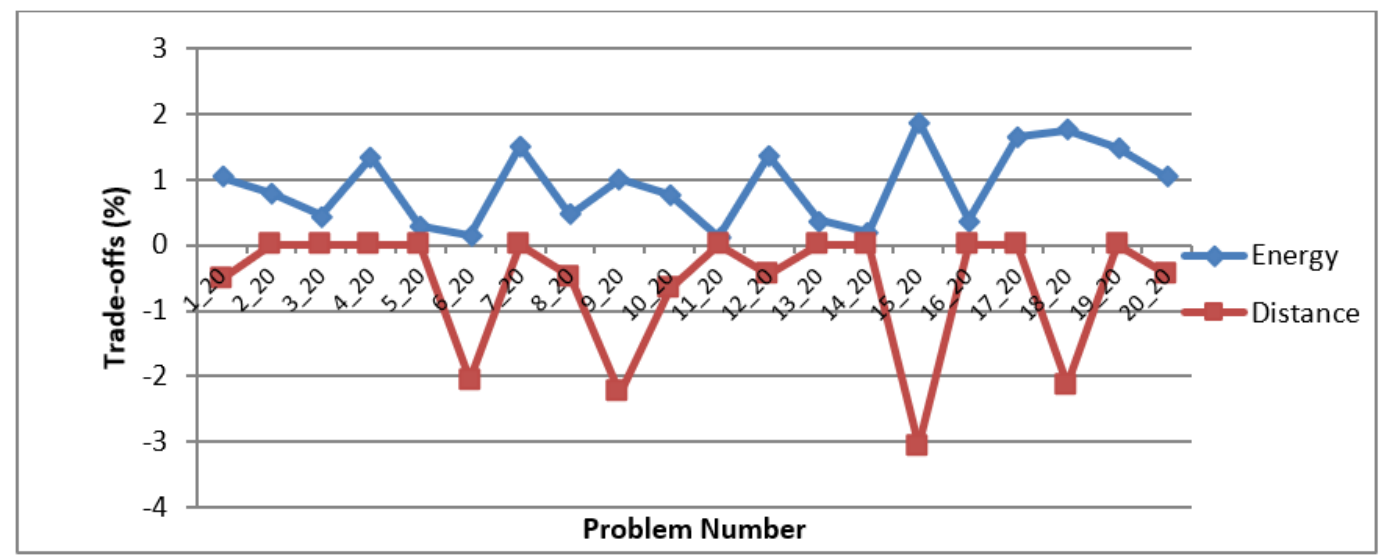

Fig. 3. Trade-offs (\%) between energy and distance.

Nowadays, energy efficient production and logistics are important issue due to energy costs. The pickup and delivery tasks are repeated actions in production logistics. Considering the fact that millions of transportation tasks will be carried out in a production enterprise, it is clear that a great energy saving will be achieved in the plants. In addition, the proposed model avoids transportation of heavy loads over long distances. It is clear that the energy saving will be much higher if the transportation area is large that has more alternative routes.

\section{Conclusions}

ATVs provide significant opportunities for the logistic industry by influencing all of the stages of the delivery and pickup tasks. This study proposes an energy saving routing and task scheduling system for delivery and pickup tasks with backhauls by using ATVs in production environments. The proposed algorithm for the initial solution minimizes the number of ATVs by considering capacity utilization rates of ATVs. Routes of ATVs and sequences of tasks are obtained by using the proposed energy saving routing and task scheduling system. The system is also compared to the traditional routing system which minimizes total distance travelled. Trade-offs between energy and distance show that the proposed system decreases energy.

Note that the costs of intra/production logistics activities have significant influence to the overall production costs. It is clear that a great energy saving will be achieved due to high energy costs and the nature of repeated transportation tasks in production plant. Future work will be concentrated on conflict free routing of ATVs for VRPB. The proposed model can be extended to cover other parameters that has effect on energy consumption of ATVs.

\section{ACKNOWLEDGEMENTS}

This work is supported by the Scientific and Technical Research Council of Turkey (TUBITAK), Contract No 116E731, project title: "Development of Autonomous Transfer Vehicles and HumanMachine Machine-Machine Interfaces for Smart Factories".

\section{References.}

Bektas, T., Laporte, G., (2011). The pollution-routing problem. Transportation Research Part B, 45, 1232-1250.

Brandao, J. (2006). A new tabu search algorithm for the vehicle routing problem with backhauls. European Journal of Operational Research, 173, 540-555. 
Chávez, J., Escobar, J., \& Echeverri, M. (2016). A multi-objective Pareto ant colony algorithm for the Multi-Depot Vehicle Routing problem with Backhauls. International Journal of Industrial Engineering Computations, 7(1), 35-48.

Dijkstra, E. W. (1959). A note on two problems in connexion with graphs. Numerische Mathematik, 1, 269-271.

Eksioglu, B., Vural A.V. \& Reisman, A. (2009). The vehicle routing problem: A taxonomic review, Computers and Industrial Engineering, 57(4), 1472-1483.

Emilio, F., Dahleh, M.A. \& Feron, E. (2002). Real-time motion planning for agile autonomous vehicles. Journal of Guidance, Control, and Dynamics, 25(1), 116-129.

Fang, K., Uhan, N., Zhao, F., \& Sutherland. J.W., (2011). A new approach to scheduling in manufacturing for power consumption and carbon footprint reduction. Journal of Manufacturing Systems, 30, 234-240.

Ghannadpour, S. and Hooshfar, M., (2016). Multi-Objective Vehicle Routing Problem with Time Windows and Fuel Consumption Minimizing. In Proceedings of 5th the International Conference on Operations Research and Enterprise Systems (ICORES 2016), 92-99.

Goetschalckx, M., \& Jacobs-Blecha, C. (1989). The vehicle routing problem with backhauls. European Journal of Operational Research, 42(1), 39-51.

Goetschalckx, M. (2011). Supply Chain Engineering (International Series in Operations Research \& Management Science), Springer, NewYork.

Herrero-Pérez, D. \& Martínez-Barberá, H., (2010). Modeling distributed transportation systems composed of flexible automated guided vehicles in flexible manufacturing systems. IEEE Transactions on Industrial Informatics. 6(2), 166-180.

Hussein, A., Mostafa, H., Badrel-din, M., Sultan, O. \& Khamis A. (2012). Metaheuristic optimization approach to mobile robot path planning. IEEE International Conference on Engineering and Technology (ICET), 1-6.

Kara, I.; Kara, B.Y., \& Yetiş, M. K., (2007). Energy minimizing vehicle routing problem. Combinatorial Optimization and Applications Proceedings. Book Series: Lecture Notes in Computer Science, 4616. 62-71.

Lin, C., Choy, K. L., Ho, G. T. S., Chung, S. H. \& Lam, H. Y. (2014). Survey of Green Vehicle Routing Problem: Past and future trends, Expert Systems with Applications, 41, 1118-1138.

Liu, S., Linbo, M. \& Jinshou, Y. (2006). Path planning based on ant colony algorithm and distributed local navigation for multi-robot systems. IEEE International Conference on Mechatronics and Automation, 1733-1738.

Magdy, Y., Shehata, O.M., AbdelAziz, M., Ghoneima, M., \& Tolbah, F. (2017). Metaheuristic optimization in path planning of autonomous vehicles under the ATOM framework. 2017 IEEE International Conference on Vehicular Electronics and Safety (ICVES), Vienna, 32-37.

Rahul, K. (2020). Robot Mission Planning using Co-evolutionary Optimization. Robotica, 38(3), 512530.

Xiao, Y., Zhao, Q., Kaku, I., \& Xu, Y., (2012). Development of a fuel consumption optimization model for the capacitated vehicle routing problem. Computers and Operations Research, 39(7), 1419-1431.

Xidias, E.K., Nearchou, A.C. \& Aspragathos, N.A. (2009). Vehicle scheduling in 2D shop floor environments, Industrial Robot: An International Journal. Vol.36 (2), 176-183. 
Xidias, E.K. Paraskevi, Z. \& Andreas, N. (2016). Path planning and scheduling for a fleet of autonomous vehicles. Robotica, 34(10), 1-17.

Xidias, E.K. (2018). On designing near-optimum paths on weighted regions for an intelligent vehicle. International Journal of Intelligent Transportation Systems Research, 17(2), 89-101.

Wang, F., Zhang, Y., \& Su, Z. (2019). A novel scheduling method for automated guided vehicles in workshop environments. International Journal of Advanced Robotic Systems, 16(3).

$\begin{array}{lr}\text { Submitted: } & 10 / 07 / 2020 \\ \text { Revised: } & 06 / 01 / 2021 \\ \text { Accepted: } & 07 / 01 / 2021 \\ \text { DOI: } \quad 10.48129 / k j s . v 49 i 1.10194\end{array}$

